\title{
Prácticas de estimulación y promoción del desarrollo infantil en la etnia mayo
}

\author{
José Angel Vera Noriega ${ }^{1}$ - Centro de investigación en Alimentación y Desarrollo, Hermosillo, México \\ Miguel Angel Torres Avila - Centro de investigación en Alimentación y Desarrollo, Hermosillo, México \\ Claudia Karina Rodríguez Carvajal - Centro de investigación en Alimentación y Desarrollo, Hermosillo, México \\ José Pablo Siqueiros Aguilera - Centro de investigación en Alimentación y Desarrollo, Hermosillo, México
}

\begin{abstract}
Resumen
En este estudio se identificaron las características de las prácticas de estimulación y promoción del desarrollo en una comunidad indígena Mayo del estado de Sonora y Sinaloa en el noroeste de México. Se evaluaron dos grupos de 50 madres cada uno: un primer grupo que llamaremos de resistencia étnica, hablaba la lengua mayo a sus hijos y participaba en un programa educativo no escolarizado cuyo objeto es estimular el desarrollo. Un segundo grupo de madres que llamaremos de asimilación étnica, no hablaba la lengua mayo y no pertenecía al programa educativo. Se realizó mediante la aplicación de entrevistas a las madres y con escalas de desarrollo psicológico para niños de 0 y 5 años de edad. Los resultados indican que la población no hablante y sin educación inicial (asimilación étnica) presenta baja frecuencia en el rango de problemas en el desarrollo y estimulación del niño en el hogar, comparado con la población hablante y con educación inicial (resistencia étnica). Se concluye en términos de un modelo intercultural de educación inicial para población indígena.

Palabras clave: Estrés de la crianza, Etnia mayo, Educación intercultural, Desarrollo del niño.
\end{abstract}

\section{Stimulation practices and promotion of child development in maya ethnicity}

\begin{abstract}
In this study the characteristics of stimulation practices and developmental practices in an indigenous Maya community from the state of Sonora and Sinaloa in the northwestern part of Mexico have been evaluated. Two groups of 50 mothers each: the first group, which we will call ethnic resistance, spoke the Mayan language to their children and participated in a non-schooled educational program of development stimulation. A second group of mothers which we will call ethnic assimilation did not speak the Mayan language and didn't belong to the initial educational program. It was accomplished by the application of interviews to mothers with the scales of psychological development for children ranging from 0 to 5 years of age. The results indicate that the non-speaking population and without the initial education (ethnic assimilation) present low frequency in the range of developmental problems and home stimulation of the child, compared to the speaking populations and with initial education (ethnic resistance). We conclude for an intercultural model of initial education for an indigenous population.

Keywords: Childrearing stress, Maya ethnicity, Intercultural education, Child development.
\end{abstract}

Actualmente se han realizado algunos estudios en zonas rurales en pobreza extrema en el Noroeste de México, enfocados en el desarrollo del niño, las practicas de crianza y salud infantil. (Vera, 2006; Vera, Calderón \& Torres, 2007). Entre los modelos de crianza propuestos, Abidin (1990); Vera y Velasco (2000); Peña y Vera (2004), relacionan las variables del ejercicio de la crianza en las madres y el desarrollo del niño, y analizan la interacción madre-hijo-padre y su asociación con estimulación del niño en el hogar. Encuentran que la variable percepción de coparticipación de la pareja se relaciona con estimulación del niño, además con interacción de los padres y también con desarrollo del niño, y por ultimo con estrés de la madre; la variable autoritarismo se vincula con estimulación del niño, interacción de los padres y con desarrollo integral del niño (Peña 2004).

${ }^{1}$ Endereço para correspondência

Departamento de Desarrollo Humano y Bienestar Social Centro de Investigación en Alimentación y Desarrollo, A.C. Carretera a la Victoria Km .6 Ejido La Victoria, Apdo. Postal 1735 - C.P. 83000 - Hermosillo, Sonora, México E-mail: psicología@ciad.mx
Sabemos que existen varios modelos para explicar la crianza y el desarrollo del niño, (Abidin, 1990; Belsky, 1984; Bronfenbrenner, 1979; León, 2005; Super \& Harkness, 1987), que basan sus propuestas teóricas en un modelo cultural que integra las percepciones, opiniones, creencias, actitudes, valores y expectativas de las madres, padres, abuelos y cuidadores involucrados con el niño en la comunidad.

Dentro de los modelos para la crianza destacan los siguientes: a) El modelo de crianza de Belsky (1984), que concluye en que la crianza como ejercicio multideterminado, dependerá directamente de las características de niño, padres y contexto social, las cuales no tendrán el mismo peso sobre el ejercicio, de manera indirecta de la personalidad de los padres y su historia comportamental; b) El modelo de crianza de Webster-Stratton (1990) supone que las condiciones estresantes tales como los eventos extrafamiliares, estresores entre los padres y estresores debido al niño, son acontecimientos en los cuales los padres tendrán que instrumentar estrategias que permitan enfrentar éstas situaciones; c) El Modelo de crianza de Abidin (1990) supone que las fuentes de estrés son aditivos multidimensionales en origen y clase; estas van desde 
eventos de vida estresantes, como la muerte de un familiar, hasta sentimientos subjetivos de las madres por sus responsabilidades de crianza; d) El Modelo de Crianza de Vera y Velasco (2000), modelo de correlación que expone algunas características de personalidad de la madre como el autoconcepto, depresión, apoyo percibido; funcionan como variables intermedias, como variables próximas la estimulación en el hogar y el estrés paterno y de resultado calidad de la alimentación, desarrollo psicológico y salud e higiene del niño; e) El Modelo de crianza Peña (2004) propone que son necesarias condiciones familiares y personales para que la madre pueda ejercer con éxito la maternidad, ya sea que funjan como facilitadoras o disposicionales, la interacción madre-hijo-padre es vista como inmediata a la promoción del desarrollo integral del niño, estrés de la crianza como disposicional y, como subyacentes el estilo de autoridad y la percepción de coparticipación del padre en la crianza.

\section{Reproducción social y crianza}

En este sentido de la reproducción, la crianza en el desarrollo infantil toma un papel importante y refiere al proceso complejo que conjunta elementos relacionados con el cuidado asistencial del niño, estableciendo normas disciplinarias, aunado a los conocimientos, actitudes y comportamientos de los padres. Además sirve de socialización entre padre hacia los hijos (Villegas, 2000). La crianza ha de cubrir aquellas necesidades relacionadas con los afectos, condiciones propias para la exploración, el aprendizaje y experiencias que estimulen el desarrollo.

Lo anterior subraya la importancia de las prácticas de crianza como procesos simbólicos vinculados a una cultura, que no tienen solamente un objetivo utilitario o de cuidado del niño sino están asociadas a las formas de reproducción social que la comunidad ha desarrollado a través del tiempo. Por ejemplo en una comunidad de clase media urbana, frente a un problema de diarrea en el niño, seguramente acudirán a un centro de salud y recibirán después de una serie de análisis clínicos, radiológicos y etc. una cantidad de medicamentos que están relacionados con la cura de esa enfermedad. En una comunidad indígena en México, aun cuando existan servicios médicos, el niño será llevado primero a un sanador, y si después de rezarle y hacerle una "limpia" no desaparecen los síntomas, se le ofrecerá algún remedio basado en la herbolaria local (Vera, 2000).

\section{Educación Inicial no escolarizada (PEINE)}

El Programa de Educación Inicial se desarrolla por parte del Consejo Nacional para el Fomento Educativo (CONAFE) fundamentalmente rurales aislados y de alta marginación en la republica Mexicana que poseen diferentes sistemas de conocimientos, normas, saberes y dones. Se sabe que en los pueblos indígenas y en las comunidades étnicas de México se tiene un idioma, patrimonio, culturas y conocimientos ricos y diversos, adquiridos de sus relaciones entre si y sus ecosistemas, que se reproducen de generación en generación y que se manifiestan a través de sus tradiciones, creencias, costumbres y ceremonias (Cunningham, 2001).

Los planes de estudio y programas de educación inicial, poseen orientaciones psicopedagógicas que estimulan el aprendizaje del español, sin prestar importancia a la diversidad lingüística y cultural de las comunidades indígenas mexicanas.

El Programa de Educación Inicial no escolarizada supone que las personas que participan adquieren conocimientos, habilidades, actitudes y valores que les permiten reflexionar sobre sus prácticas de crianza y que las decisiones que tomen sobre estos aspectos puedan mejorar el desarrollo del niño y de la vida familiar.

La educación inicial tiene como objetivo brindar asesoría a los padres, madres y a las personas que participan en el cuidado de la crianza de los niños en las comunidades rurales e indígenas de alta marginación, con el fin de enriquecer las prácticas de crianza que favorezcan el desarrollo de competencias y lograr, entre otros beneficios, una transición exitosa a la educación preescolar (SEP, 2004). Se desarrolla de forma no escolarizada y promueve la participación de la familia de la comunidad en la que se desarrolla además de contribuir en el desarrollo integral de los niños, tomando en cuenta su crecimiento social, afectivo y cognitivo, la expresión oral y artística, la psicomotricidad y el respeto de sus derechos, en donde el Estado asume el compromiso y la responsabilidad de atender sus necesidades y de brindar una educación preescolar con calidad (SEP-CONAFE, 1999).

El modelo de educación inicial no escolarizada, tiene como objetivo enriquecer las practicas de crianza que favorezcan el desarrollo de competencias y lograr una transición exitosa a la educación preescolar, dicho modelo se compone de 4 ejes curriculares que cubren las necesidades básicas y de desarrollo de los infantes: 1) Cuidado y protección infantil (salud, alimentación, higiene y protección); 2) Personal y social (identidad, autoestima, autorregulación, autonomía e interacción con otros); 3) Lenguaje y comunicación (a través de gestos, sonidos y movimientos, con palabras, frases, oraciones y números, y gráfico platica); 4) Exploración y conocimientos del medio (control y equilibrio del 
cuerpo, exploración y manipulación de objetos, representaciones y categorización).

Dentro de este modelo, los adultos encargados del cuidado de los niños de 0 a 5 años (población objetivo), deberán desarrollar competencias específicamente relacionadas con los ejes curriculares planteados para esas edades, las competencias esperadas, están enfocadas a conductas de tipo social. El programa de educación inicial no escolarizada funciona mediante un promotor educativo que ha de poseer ciertas habilidades y competencias y vivir en la comunidad en la que labora con el programa (SEPCONAFE, 1999). El papel del Promotor Educativo, es de suma importancia para que el proyecto tenga efectividad, tiene como finalidad brindar el apoyo a los padres de familia y comunidad en su labor educativa. Su función es establecer la relación directa y cotidiana con los padres y miembros de la comunidad, y convencerlos de que los procesos del desarrollo del niño más importantes tienen que ver con la inteligencia, el pensamiento y el lenguaje.

Este programa se propone contribuir en el fortalecimiento de las capacidades de las personas en sus contextos socioculturales, que gocen de la posibilidad de acceder a las oportunidades que se les presenten en términos de salud, educación, empleo entre otros (PNUD, 2001). Como lo mencionó Sen (2000), un desarrollo del cual el objetivo es dotar a las personas de las capacidades necesarias para tener realizaciones que les reporten satisfacción, que la vida de las personas sea larga y de calidad, que los infantes logren un adecuado desarrollo psicológico y físico, lo que significa tener acceso a recursos de salud y educación. Se trata de un concepto educativo muy amplio, pero que se encuentra poco estructurado y fue diseñado sin la participación de las comunidades y es manejado por líderes locales que utilizan criterios de exclusión e inducción al programa, que ha trasformado al PEINE en las comunidades indígenas en un factor más de inequidad y desigualdad.

\section{Modelo Intercultural de Educación Inicial}

En el informe sobre el desarrollo humano de los pueblos indígenas en México (2006), se mencionó que los grupos indígenas mostraban indicadores sociales rezagados con respecto al resto del país, lo cual llama a acciones concretas para entender las causas y actuar con intervenciones públicas específicas. Existen en Sonora doce regiones geográficas con poblaciones indígenas entre las cuales destacan en el sur del Estado dos regiones con un alto nivel de pobreza, siendo la mayoría comunidades rurales, donde se asienta y reside la etnia Mayo, agrupando los municipios de Bacum, Cajeme, Etchojoa, Huatabampo, Navojoa y San
Ignacio Río Muerto (Camberos \& cols., 1994). En estas comunidades el Instituto Nacional de Estadística, Geografía e Informática (INEGI, 2005) señaló que la mayoría de las madres y padres sólo alcanzaban a cursar como máximo 6 años de educación y la lectoescritura no es una actividad de importancia en sus vidas cotidianas, ya que el alimento se convierte en el elemento central de todo tipo de aspiración y planeación. De acuerdo con los indicadores educativos, en Sonora se presentó una deserción escolar indígena mayor en 2.2 puntos porcentuales con respecto a la media nacional que es de 1.3. El indicador de reprobación para el modelo educativo bilingüe tuvo un puntaje mayor en 3.1\% al que obtiene el estado y en $1.0 \%$ con respecto al nivel nacional (SEP, 2004).

Los servicios de educación indígena en el Noroeste de México, atienden a niñas y niños hablantes de la lengua indígena en educación inicial. Éstos están distribuidos en 20 zonas de supervisión, 85 escuelas, 2149 alumnos y 88 maestros. Los servicios de educación indígena, en el estado de Sonora, atienden a seis lenguas indígenas, una de ellas la lengua Mayo con 1386 niños en educación inicial (SEP, 2004).

Un modelo sociocultural de educación inicial deberá mezclar las aspiraciones de un mundo global con las prácticas de los pueblos indígenas, en donde las inversiones en educación inicial y preescolar, mejoren las capacidades futuras, promoviendo una preparación afectivo social adecuada en la niñez que contribuya a las habilidades de los adultos para ganarse la vida, ser económicamente productivos y respetar los valores para la vida armónica en sociedad (Vera, 2004).

El conocimiento sobre cómo la cultura, el contexto de la familia y los padres influyen en el desarrollo de ciertas dimensiones de la personalidad de los niños, resulta especialmente valioso para determinar qué tipo de acciones y relaciones entre padres e hijos resultan pertinentes y relevantes para fomentar el desarrollo de la madurez, la conducta competente y la responsabilidad social en los niños. Este conocimiento puede permitir que los padres logren con mayor probabilidad, contribuir a un desarrollo psicológico sano de sus hijos. Estudiar los procesos de crianza en la etnia Mayos en Sonora aportaría una visión amplia para en un futuro contar con la información pertinente y relevante para modificar el programa de Educación Inicial no escolarizada.

Los padres de áreas rurales con pobreza extrema, limitan la educación de los niños, decidiendo las inversiones de capital familiar tanto monetarias, como de recursos de tiempo y afectivo-emocional a la búsqueda de alimentos, vestido y techo (Vera, 2003). Se pueden advertir dos grandes procesos de reproducción social en las comunidades Mayo, por un lado las que 
están vinculadas a conservar la posición en la estructura social y económica a través de la transmisión del patrimonio familiar en forma directa y/o indirecta de una generación a otra y por otro lado la conservación de la cultura, tradiciones, mitos y saberes.

El estudio de los procesos de reproducción social a través de la crianza permiten obtener la información necesaria sobre las variables que obstaculizan y/o facilitan este ejercicio, tomando en cuenta por un lado la forma como ésta se relaciona con la preservación de la cultura y por otro para documentar los procesos sociales de aprendizaje y las estrategias familiares para promover el razonamiento lógico, moral y estético. Son tres las condiciones necesarias para la crianza: un contexto libre de presiones, un nivel de desafío practicando el conflicto positivo a través del recurso didáctico de la frustración optima y finalmente la mitología y ritos que la comunidad requiera preservar (Arranz, 2002).

Las variables que intervienen en la crianza son el soporte social, familiar y comunitario, la maternidad paternidad, relación de pareja y bienestar personal, la percepción de bienestar de la madre en la crianza, la calidad de estimulación, el desarrollo integral y habilidades cognitivas del niño. Por lo que el desarrollo definido como un proceso multifacético que además de su multidimensionalidad, posee un carácter incluyente, donde el ser humano como tal, pasa a través de diferentes etapas y en esta transición se desarrollan las diferentes habilidades integrales que permiten fortalecer la autonomía del niño para integrarse en su ambiente familiar y social, y desarrollar habilidades como las habilidades en el área motora fina, motora gruesa, sensocognitiva, socioafectiva, lenguaje y hábitos (Shaffer, 2000). En ese sentido, los niños que gozan de aceptación social tienden a responder con mayor sensibilidad a las interacciones comenzadas por otro; son atentos a las verbalizaciones, siguen sugerencias, aprueban, imitan, toman iniciativas y comunican lo que quieren (Jaramillo y cols., 2006).

La comunidad indígena Mayo "El Jupare", se encuentra al sur del estado de Sonora, en el municipio de Huatabampo. En la zona norte del estado de Sinaloa se ubican, las comunidades rurales como El Ranchito de Mochicahui, La Cruz Pinta y El Carricito, El Fuerte, Sinaloa, en los cuales habitan 500 familias. En este artículo se estudian las variables mediadoras de estrés de la crianza, y las próximas, interacción padre-hijomadre, la estimulación del niño en el hogar, y finalmente la relación de éstas en el desarrollo integral del niño. Se pretende contribuir al mejoramiento del programa de Educación Inicial no escolarizada, estudiando su currículo y contrastándolo con los resultados de esta investigación, para determinar su pertinencia, y a partir de ello, realizar ajustes necesarios y propuestas para adaptarlo a la comunidad, partiendo de las condiciones de aquellos a quienes va dirigido el programa, en este caso la comunidad indígena mayo.

\section{Metodología}

La elección de la población para el estudio, fue de tipo muestreo no probabilístico intencional (Kerlinger \& Lee, 2002), por medio de visitas a 3 comunidades, en el municipio de El Fuerte, Sinaloa y, 4 más en el municipio de Huatabampo, Sonora, ambos estados pertenecientes al Noroeste de México. La muestra estuvo conformada por 100 madres, cada una con un hijo de entre 1 y 5 años de edad, elegidas al azar, a través de un procedimiento sin reemplazo, utilizando una urna para las comunidades cercanas a Mochicahui y mediante el método de barrido para las comunidades cercanas a el Júpare. El estudio fue de tipo no experimental, ex post facto, descriptivo y transversal correlacional, para conocer las variables que intervienen, en la crianza y desarrollo del niño, así como establecer relaciones entre las variables. De la muestra, el $52 \%$ de las madres era mayor de 28 años, de los niños, el 50\% estaba bajo el programa de educación inicial no escolarizado y la madre hablaba y transmitía la lengua nativa y el otro $50 \%$ no estaba en el programa y no hablaban la lengua, de los niños el 50\% pertenecía al sexo femenino y el 50\% al masculino. Se trata de un grup familiar con un alto grado de identidad promotor de mitos, ritos y lenguaje, interesado en transmitir a la generaciones futuras su un patrimonio cultural y de un segundo grupo occidentalizado compuesto por familias que ya no trasmiten la lengua a sus hijos y no participan del programa de Educación Inicial.

\section{Los Mayo-Yoreme, etnia del Noroeste de México}

Los Mayo se dedican a la agricultura, a la cría de ganado vacuno, lanar o caprino y a la pesca (Aguilar, 2007), en jacales de adobe con techo de tierra y una ramada al frente de la vivienda, al centro hay una ramada con techo de palma donde realizan ceremonias y ritos religiosos propios de la familia. Las mujeres Mayo se dedican a la confección de cobijas de lana utilizando un telar vertical y lana de los borregos de sus rebaños. Al encontrarse territorio Mayo cerca del mar, algunos de los mayos pescan con redes desde la orilla (CNDPI, 2006). En la práctica religiosa los Mayos retoman la lengua, la industria y las tradiciones y es aquí donde se desenvuelve la verdadera vida comunitaria de los Mayos sin permitir la entrada de los blancos. El nacer dentro de la familia Mayo no es condición suficiente para el autoreconocimiento pero sí 
para el heteroreconocimiento, por ese sólo hecho los otros Mayos reconocen al niño como parte de la etnia (Aguilar, 2007). En este pueblo indígena se desarrolla una complicada red de relaciones sociales dirigidas a explicar el origen y la pertenencia a un grupo determinado, así como también explicar cuáles son los procesos que transformaron durante el tiempo su propia cotidianidad. La existencia de lugares sagrados ha permitido no solo delimitar fronteras geográficas, sino también explicar los orígenes de los mitos de creación y dándole un sentido de identidad al grupo (Rojas, 2002).

\section{Instrumentos de medida}

Los instrumentos que se utilizaron fueron los siguientes: a) Escala de desarrollo integral (EDIN de Atkin y cols., 1987), el cual proporciona información detallada sobre el desarrollo del niño, en 6 categorías, motricidad gruesa, motricidad fina, sensocognitiva, lenguaje, socioafectiva y hábitos; b) Índice de estrés parental (IEP de Abidin, 1983), que provee información de las características de la madre y el niño que pueden convertirse en una fuente de estrés, y por lo tanto contribuir a la modificación de la conducta materna en el continuo funcional-disfuncional; c) Estimulación del niño en el hogar (HOME de Caldwell \& Bradley, 1968) mide la estimulación del niño en el hogar en 6 subescalas, responsabilidad emocional y verbal de los padres, aceptación de la conducta del niño, organización del medio ambiente físico, provisión de materiales de juego, si los padres se involucran con el niño y las oportunidades de variedad en la estimulación; d) Interacción Madre-hijo-padre (Peña, 2004), nos arroja información sobre el tiempo que los padres pasan con el niño, las actividades que realizan, juguetes que le proporcionan y el comportamiento que estos tienen en presencia del niño; e) Inventario de alianza para la paternidad (Abidin \& Brunner, 1995) evalúa el grado de coparticipación y compromiso que los padres perciben de su conyugue, además de describir la parte de la relación de pareja en relación a la crianza; f) Escala de Estilo de Autoridad de la madre de Vigano y Diaz-Loving (1990) ajustada por Vera y Montiel (1998), que tiene como objetivo que la madre asuma la evolución de su conducta, en relación con el niño.

Para complementar la información se utilizó una Hoja de Identificación, la cual integró, datos específicos de niño y la madre, del padre solo la edad y ocupación, también se integraron datos referentes a enseres e infraestructura del hogar. Después de validar los instrumentos en comunidades rurales del Estado de Sonora, se trabajó en 2 fases, se entrenó a los entrevistadores en la primera y en la segunda, ya en las comunidades, se localizó a las madres y fueron entrevistadas en varias ocasiones, una vez que se les explicó el motivo de la visita y estas firmaron un consentimiento informado para participar en la investigación. Para la captura y manejo de los datos, se utilizó la versión 15 del SPSS.

\section{Resultados}

La distribución de la población, fue equitativa para hablantes y no hablantes de la lengua Mayo, para usuarios del programa de educación inicial y aquellos que no lo eran. Para el EDIN, se colocaron los datos según los rangos de edad que van del 1 a 2 , de 2 a 3 , de 3 a 4 y de 4 a 5 años de edad correspondientemente, para evitar rangos medios entre las edades y evitar cualquier tipo de confusión, tomando en cuenta los rangos ya establecidos por Peña (2004).

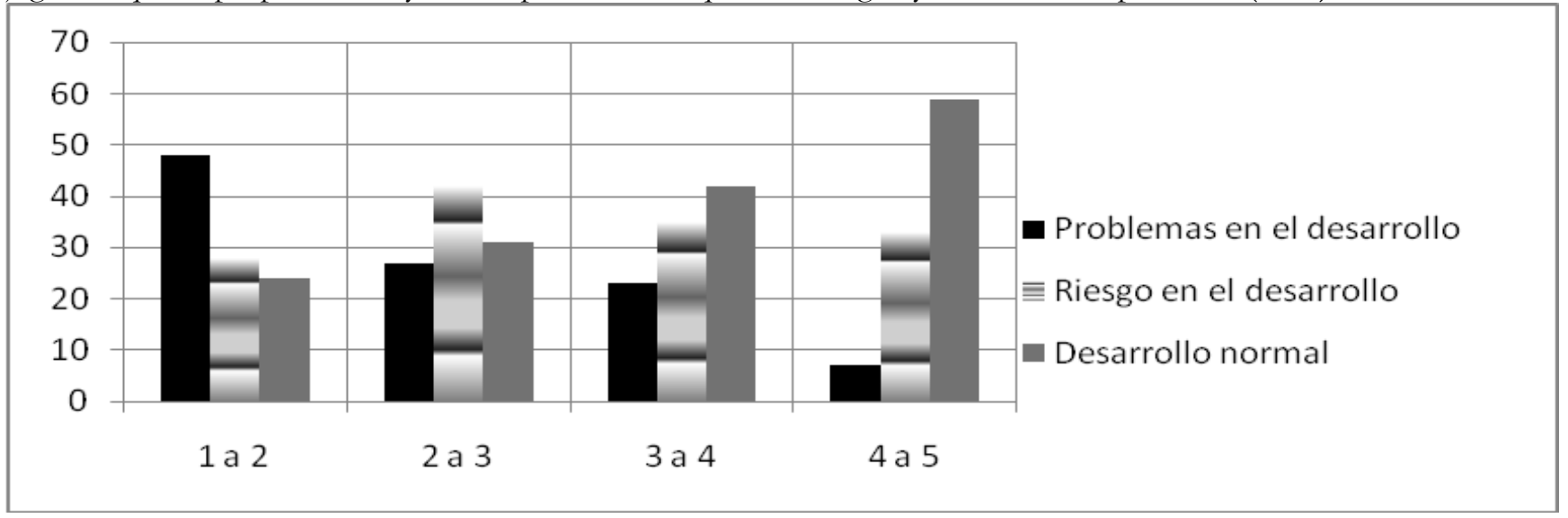

Figura 1 - Datos porcentuales de los resultados de la medida de evaluación del desarrollo (EDIN) para una muestra de Madres hablantes con educación inicial 
En la Figura 1 se observa que los niños de madres que hablan la lengua y asisten al programa de educación inicial del gobierno federal, presenta un porcentaje mayor de niños con problemas y en riesgo. Para elaborar la Figura 1 y 2 cada niño es ubicado como positivo o negativo (0-1) en cada una de las áreas de evaluación: motora fina, gruesa, senso perceptual, lenguaje, socializaciones y hábitos. O sea cada niño contribuía una sola vez según su ejecución en la prueba colocándose en cada una de las seis áreas en solo uno de los tres niveles de calificación: normal, riesgo y problema.

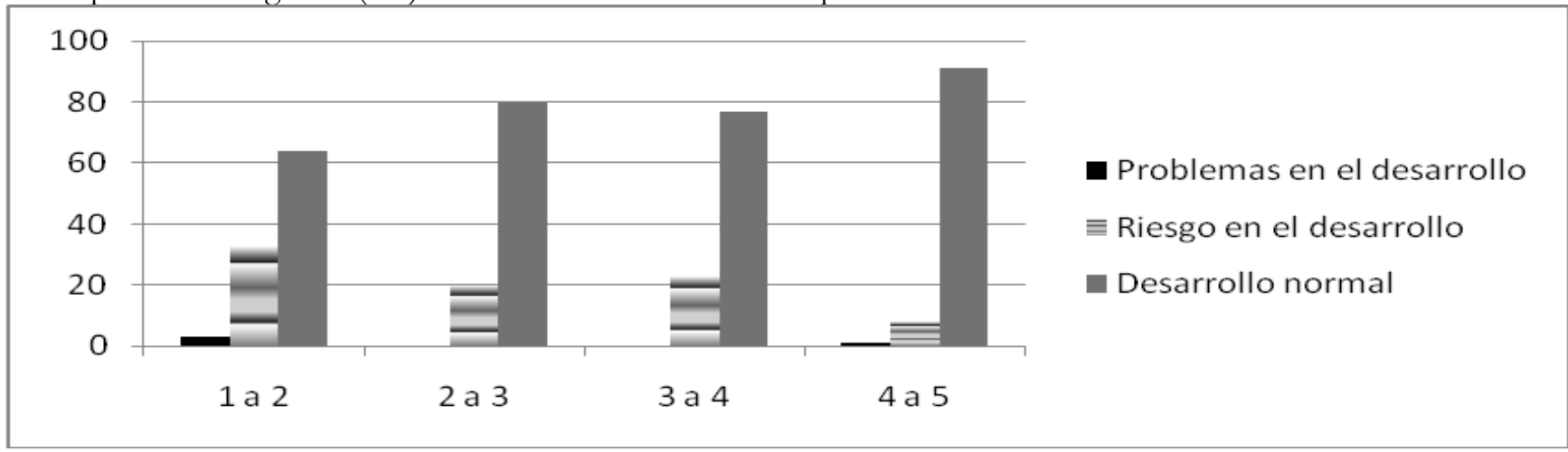

Figura 2 - Datos porcentuales de los resultados de la medida de evaluación del desarrollo (EDIN) para una muestra de madres no-hablantes sin educación inicial

La Figura 1 nos presenta un alto porcentaje de niños de uno a cuatro años con problemas en el desarrollo y otros tantos en riesgo. Por otro lado, el grupo de madres e hijos que no hablan ni transmiten la lengua y no asisten al programa, tienen niños con muy pocos problemas y riesgo en el desarrollo.

Para el HOME, fue necesario reagrupar los puntajes obtenidos según los valores resultantes en la estandarización que realizó Peña (2004) en la zona rural de Sonora en donde para obtener los puntajes se realizaron percentiles de los grupos dando como resultados puntajes de estimulación inadecuada, moderada y adecuada.
En la Tabla 1 se colocó el porcentaje de niños que se ubicaron según su puntaje en inadecuados, moderados o con estimulación adecuada, separando por un lado los niños con madres que hablan y enseñan la lengua y por otro lado los que tienen madres que no la hablan ni la enseñan, observando a su vez cómo es que los porcentajes más altos se encuentran en estimulación inadecuada en todas las dimensiones los obtienen los niños con madres hablantes de la lengua que a su vez están bajo el programa de educación inicial.

Tabla 1 - Estadígrafos obtenidos según el tipo de estimulación que recibe el niño en el hogar, diferenciando a la población hablante de los no hablantes de la lengua Mayo

\begin{tabular}{|c|c|c|c|c|c|}
\hline Dimensión & $\mathrm{X}$ & DS & Estimulación & $\%$ Hablantes & $\%$ No Hablantes \\
\hline \multirow[t]{3}{*}{ Respuesta verbal y emocional } & 8.73 & 2.29 & Inadecuada & 32 & / \\
\hline & & & Moderada & 20 & 46 \\
\hline & & & Adecuada & 48 & 54 \\
\hline \multirow[t]{3}{*}{ Organización del medio ambiente } & 4.31 & 1.17 & Inadecuada & 42 & 14 \\
\hline & & & Moderada & 42 & 68 \\
\hline & & & Adecuada & 16 & 18 \\
\hline \multirow{3}{*}{$\begin{array}{l}\text { Provisión y funcionalidad de } \\
\text { materiales de juego }\end{array}$} & 4.19 & 2.80 & Inadecuada & 88 & 10 \\
\hline & & & Moderada & 12 & 68 \\
\hline & & & Adecuada & / & 22 \\
\hline \multirow{3}{*}{$\begin{array}{l}\text { Los padres se relacionan con el } \\
\text { niño }\end{array}$} & 4.68 & 2.19 & Inadecuada & 30 & / \\
\hline & & & Moderada & 32 & 6 \\
\hline & & & Adecuada & 38 & 94 \\
\hline \multirow[t]{3}{*}{ Oportunidad para la variedad } & 3.12 & 1.04 & Inadecuada & 4 & 2 \\
\hline & & & Moderada & 68 & 58 \\
\hline & & & Adecuada & 28 & 40 \\
\hline
\end{tabular}


Tabla 1 - Estadígrafos obtenidos según el tipo de estimulación que recibe el niño en el hogar, diferenciando a la población hablante de los no hablantes de la lengua Mayo

\begin{tabular}{llrrcc}
\hline Dimensión & $\mathrm{X}$ & $\mathrm{DS}$ & Estimulación & \% Hablantes & \% No Hablantes \\
\hline Total & 26.12 & 6.77 & Inadecuada & 68 & 6 \\
& & & Moderada & 30 & 92 \\
& & & Adecuada & 2 & 2 \\
\hline
\end{tabular}

En cuanto al manejo de la autoridad de las madres en la zona rural indígena se obtuvieron valores de media y desviación estándar por separado para cada una de las poblaciones, por un lado los hablantes de la lengua y por otro lado los que no la hablan. La Escala de ejercicio de autoridad de la madre, derivado de la Escala Multidimensional de Autoritarismo en la Cultura Mexicana, tiene una media mínima a obtener de 26 y un máximo de 130. Con este dato podemos describir por un lado a las madres hablantes de la lengua que obtuvieron una media de 72.58 , y por otro lado las madres no hablantes con una media de 78.80; los dos grupos superaron el valor medio que es de 65 puntos, representándolas como madres que utilizan frecuentemente técnicas de enseñanza-aprendizaje basadas en la evitación y el escape y utilizan el castigo para mantener a sus hijos sujetos a las normas, y reglas de la familiar y comunidad.
Evaluando el grado de coparticipación y compromiso que los padres perciben en su conyugue en el IAP (Inventario de Apoyo Paterno), se llevo a cabo un Alpha de Cronbach, y el índice para todo el inventario fue de 0.779 . Se seleccionaron aquellos reactivos que mediante un análisis factorial resultaron con los mayores pesos factoriales $(\geq .40)$ y que a su vez resultaron estar relacionados con aspectos que incluyen sentimientos y actitudes que requieren que los padres mantengan comunicación y acuerdos permanentes.

En el IEC (Índice de Estrés de la Crianza), se aprecia que los valores de media para los dos grupos (hablante y no-hablante) son similares, las diferencias se pueden encontrar en las desviaciones estándar y en la dimensión de competencia en la madre con un valor de significancia de .011 (ver Tabla 2).

Tabla 2 - Resultados del análisis de varianza para las dimensiones del instrumento, Índice de Estrés de la crianza para las madres hablantes y no hablantes de la lengua Mayo

\begin{tabular}{llcccccc}
\hline & Dimensiones & \multicolumn{3}{c}{ Hablante } & \multicolumn{2}{c}{ No Hablante } \\
\hline \multirow{3}{*}{ Temperamento } & Distractibidad & .592 & $\mathrm{P}$ & $\mathrm{X}$ & $\mathrm{DS}$ & $\mathrm{X}$ & $\mathrm{DS}$ \\
\cline { 2 - 7 } del niño & Humor & .631 & .529 & 23.28 & 4.92 & 22.78 & 3.37 \\
& Adaptabilidad & -1.64 & .103 & 26.88 & 5.83 & 11.80 & 3.14 \\
& Demanda & .637 & .504 & 10.80 & 2.64 & 28.66 & 4.95 \\
Competencia & Distractibidad & 2.59 & .011 & 17.30 & 2.88 & 16.46 & 2.41 \\
de la madre & Humor & -1.38 & .170 & 12.40 & 2.61 & 13.04 & 1.84 \\
& Adaptabilidad & -.55 & .584 & 17.46 & 4.31 & 17.86 & 2.79 \\
& Demanda & .183 & .855 & 11.70 & 2.58 & 11.62 & 1.68 \\
\hline
\end{tabular}

Las correlaciones más altas entre las variable del IEC (población hablante y bajo el Programa educativo), las encontramos entre la de demanda y distractividad $\left(.500^{* *}\right)$, competencia y salud $\left(.483^{* *}\right)$, para la subescala de competencia de la madre. En la subescala del niño tenemos las más altas entre humor y adaptabilidad (.299*); y en las variables que están asociada a las dimensiones de la madre y el hijo es adaptabilidad-depresión (.400**) (ver Figura 3).

En la Figura 3 de correlación para la población No-hablante de la lengua y que no están bajo el
Programa de Educación Inicial, se encuentran relaciones similares a las anteriores entre las variables como "demanda" relacionada con "distractibilidad" $\left(.368^{* *}\right)$ y "distractividad" con "demanda" $\left(.316^{*}\right)$, pero también encontramos relaciones entre "humor" y "demanda" (.425**); por otro lado para las dimensiones de la madre correlacionan "depresión" y "salud" (.322*); y las variables que están asociada a las dimensiones de la madre y el hijo es "humor" y "apego" (.334*). 

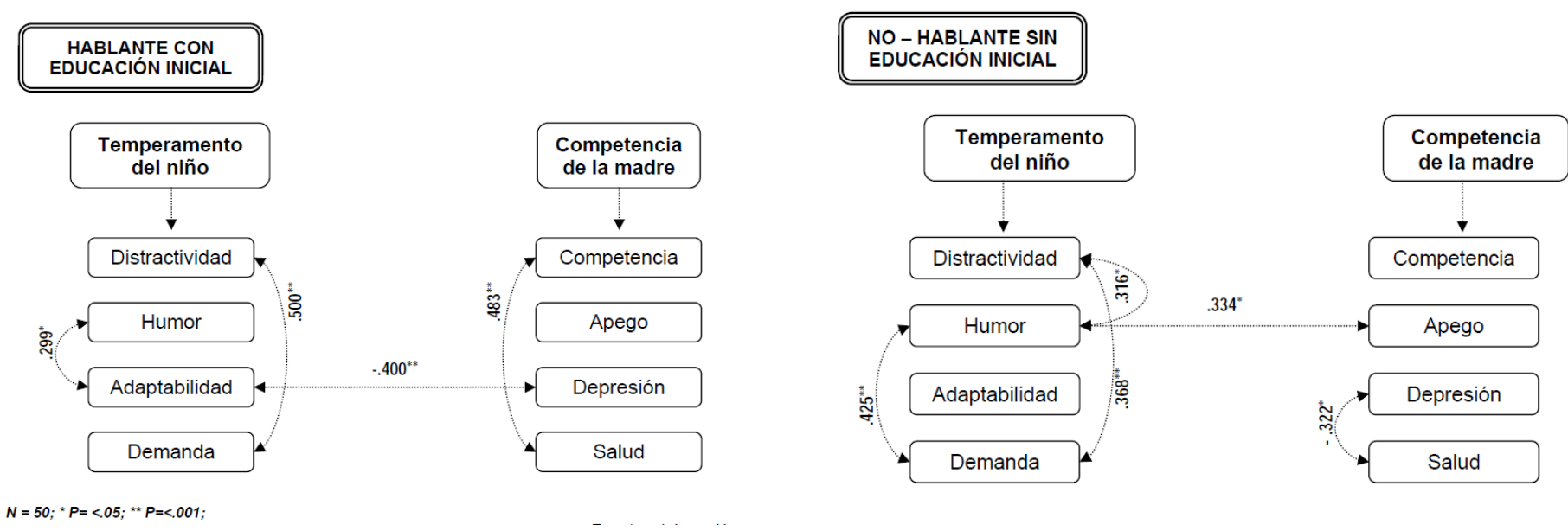

$N=50 ;{ }^{*} P=<.05 ;{ }^{\star \star} P=<.001$

Figura 3 - Correlación entre variables resultantes del inventario de Estrés parental para la población Hablante y no hablante de la lengua Mayo con educación inicial y sin educación inicial

Las correlaciones significativas para el HOME, se encontraron sólo para la población hablante de la lengua y el programa de educación inicial. La "provisión de materiales de juego", se asocia con las "respuestas verbales y emocionales" (.352*), con la “organización del medio ambiente" (.306*), y la "relación de los padres con los niños" $(.339 *)$ (ver Figura 4). Para la población no-hablante ninguno correlaciono significativamente, están basándose en diferentes técnicas para estimular al niño.

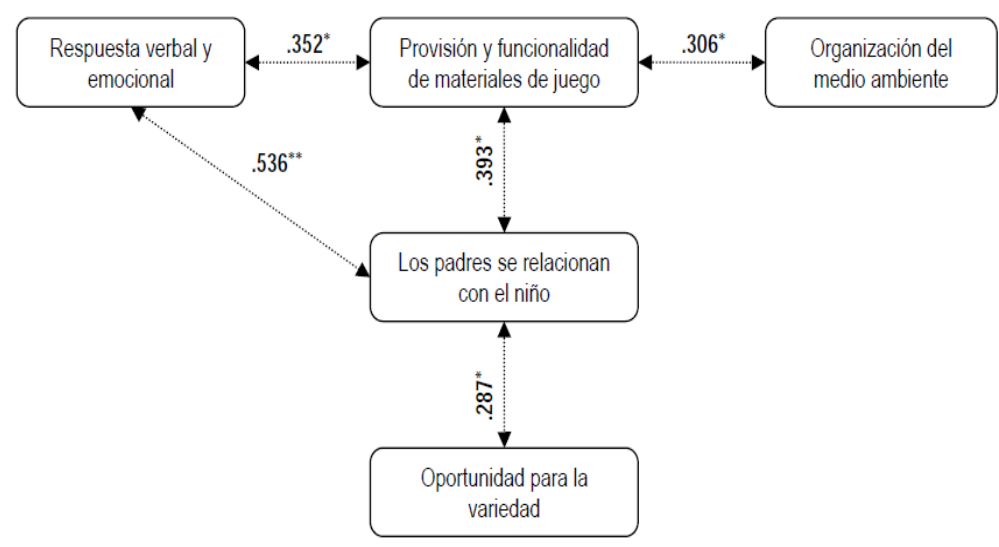

$N=50 ;{ }^{*} P=<.05 ;{ }^{\star \star} P=<.001 ;$

Figura 4 - Correlación de variables de las dimensiones del inventario de Estimulación del niño en el hogar (HOME) para la población hablante de la lengua Mayo con educación inicial

La correlación para las variables de la escala del desarrollo integral del niño en el hogar para la población hablante de la lengua y que está dentro del programa de educación inicial, destaca la asociación de la variable "lenguaje", para los hijos de madres hablantes de la lengua y que está bajo el programa de educación inicial. El "lenguaje" correlaciona de manera directa con la variable "motora gruesa" (.392**), "motora fina" (310*), "sensocognitiva" (.500**) y "socio afectivo" (.359*) y "socio afectiva" correlaciona con "hábitos" (.627**). Por otro lado, para la población no-hablante de la lengua y que no está bajo el programa de educación inicial, el "lenguaje" correlaciona con la mayoría de las áreas de desarrollo: "motora gruesa" 
(.499**), "sensocognitiva" (.302*) y "socio afectivo" $\left(.313^{*}\right)$ y esta ultima correlaciona nuevamente con "hábitos" (.405*) (ver Figura 5). Lo que se puede resaltar de esta correlación es como la variable "motora fina”, para esta población es la única que no correlaciona con "lenguaje", ni con ninguna de las otras variables propuestas por la escala.

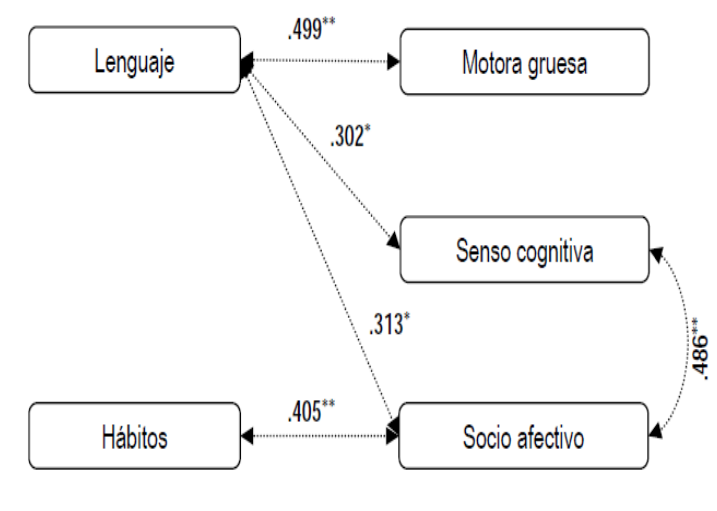

$N=50 ;{ }^{*} P=<.05 ;{ }^{* *} P=<.001 ;$

$N=50 ;{ }^{*} P=<.05 ; * * P=<.001$

Figura 5 - Correlación de variables del inventario del Desarrollo Integral del Niño en el hogar para la población hablante de la lengua Mayo con educación inicial y no-hablante de la lengua Mayo sin educación inicial

\section{Discusión}

Los resultados obtenidos en la escala de desarrollo integral del niño (EDIN), entre la población no-hablante y que no está dentro del programa de educación inicial, presenta una baja frecuencia de niños en el rango de problemas en el desarrollo, en comparación con la población hablante y que está bajo el programa de educación inicial.

Tomando en cuenta los rangos de la estandarización realizada en una población rural con pobreza extrema del Estado de Sonora (Peña, 2004), y volviendo a los datos, se esperaría encontrar a la población hablante y que está dentro del programa de educación inicial no escolarizada con menos problemas en el desarrollo, quizás podríamos argumentar que es el mismo programa el que causa un efecto de contraste en los niños, ya que las condiciones culturales a las que ellos han estado expuestos son distintas y por lo tanto sus expectativas de la crianza generan distintos logros con interpretaciones diferenciales contrastantes que el programa de educación inicial no considera en su formato universal y único. Pérez-Gómez, (2008) muestra que los indígenas Mayo del norte de Sinaloa recibían una estimulación adecuada y moderada en más del $50 \%$ de los participantes.

En la escala de estimulación del niño en el hogar (HOME), son los niños que tienen madres hablantes de la lengua y que están bajo el programa de educación inicial quienes obtienen los porcentajes de población más elevados en estimulación inadecuada, en contraste con los niños que tienen madres no hablantes de la lengua y que no están bajo el programa de educación inicial.

El instrumento de índice de estrés de la crianza, el aspecto más importante está en las dimensiones de distractibilidad y demanda para las dos poblaciones por igual. El resultado más valioso en la crianza es el desarrollo de los valores y premisas sociohistórico-culturales en donde el desarrollo cognitivo y psicológico juega un papel de facilitador y el desarrollo socio-moral del niño es determinante. El uso del enojo, la vergüenza y el miedo como ejercicio socio-moral de reconocimiento de valores, rituales y premisas es una práctica recurrente en la familia indígena para lograr que los niños lleguen a convertirse en "personas listas". Para los niños, el "ser listo" implica no sólo la preparación cognitiva que lo vuelva competente en su ambiente y lo prepara para el futuro. Para los indígenas "ser listo" implica esencialmente el desarrollo sociomoral, entendimiento de reglas y normas de actuación en un mundo que se torna distinto y está asediado por una sociedad que lo asimila y lo homogeniza (León, 2005).

Las diferencias en cuanto a las prácticas de crianza cuando se habla la lengua Mayo y cuando no se habla, las podemos encontrar en el inventario de Estrés parental, en donde son las madres del Jupare hablantes de la lengua mayo y que están bajo el programa de educación inicial, quienes adoptan una actitud de competencia delante de la salud (.483**) y dentro del temperamento del niño la variable demanda delante de 
la distractividad (500**). Lo anterior contrasta con los resultados de Laborin y Vera (2006), quienes reportaron que las madres adoptan una conducta propia ante las exigencias y demandas del niño. Pero esto a diferencia de las madres de Mochicahui no hablantes de la lengua y que no están dentro del programa de educación inicial se observaron diferencias de relaciones con la variable "humor" ya que esta última a diferencia de la otra población hablante y con programa de educación inicial, se relaciono con "distractividad" $\left(.316^{*}\right)$ con "apego" $\left(.334^{*}\right)$ y con "demanda" (425**).

Dentro del inventario de estimulación del niño en el hogar (HOME) la población hablante de la lengua mayo y que está dentro del programa de educación inicial encontramos relaciones entre la variable "provisión y funcionalidad de materiales de juego" con las demás variables como "respuesta verbal y emocional" (352*) "organización del medio ambiente" $\left(306^{*}\right)$ y "los padres se relacionan con el niño" (393*).

La provisión y funcionalidad de materiales de juego está relacionada con las competencias dentro del programa de educación inicial no escolarizada y las madres hablantes del programa se capacitan para estimular las diferentes áreas y favorecer el desarrollo del niño en diferentes ámbitos (SEP-CONAFE, 1999). A diferencia de la población no hablante de la lengua mayo y que no está dentro del programa de educación inicial no escolarizada quienes no correlacionaron en ninguna de las áreas del inventario de estimulación del niño en el hogar, por falta de este entrenamiento, pero a demás porque siguen el patrón de estimulación que heredaron de sus padres. No tratan de provocar intencionalmente el contacto del niño con un objeto que promueva lenguaje o pensamiento, sino que permiten libertad al niño para que el descubra y desarrolle sus contactos.

\section{Conclusiones}

Las madres de tiempo completo de la zona rural, comparadas con madres de la zona urbana, estimulan de manera significativa el desarrollo del niño obteniendo una asociación positiva con la estimulación del niño (Vera \& Montiel, 1998; Vera \& Peña, 2005). Se observa que en la medida que los niños crecen, y adquieren mayor independencia, sus puntajes en desarrollo se ven favorecidos, debido a los procesos de socialización que corren de manera secuencial: primero al lactante en infante hasta los dos años se le restringe el espacio de actuación al entorno materno que lo mantiene en corralitos cerca de ella y seguido después de los dos años comienza un proceso de liberación hacia los amplios espacios de la comunidad en donde la familia extensa da cuenta de su conducta lúdica y social y la comunidad entera se responsabiliza de la seguridad del niño.

Las madres no reportan tener mayores dificultades en la crianza del niño, con el manejo y control aún cuando reportan utilizar castigos leves, regaños y reforzamiento negativo. Se observa que existe libertad de movimiento dentro de la comunidad después de los 2 años, monitoreado de cerca por la madre o familia quienes suponen que poco a poco el niño es "listo" y este lo hace consciente de su actuación (León, 2005).

En lo referente al uso de la lengua indígena Mayo, los niños de la tercera generación en la comunidad tienen madres que aún cuando hablan la lengua, no lo promueven posiblemente en 15 años muy pocas personas hablarán Mayo. En las escuelas bilingües de la región los maestros (as) no hablan la lengua y si la hablan no la enseñan. Además, algunas madres colocan al Mayo como lengua de poco prestigio consideran que hablarles en Mayo interfiere con el aprendizaje del español, por lo cual esperan que el niño cumpla 5 o 6 años para hablarle en la lengua. Las madres suponen existe una relación directa proporcional entre el dominio del Mayo y la incapacidad de expresión en español, y por tanto de éxito en el mundo occidental. Las maestras bilingües por su lado apoyan esta creencia y piden a las madres que no les hablen en Mayo en sus casas.

Vera, Calderón y Torres (2007), desde una mirada cualitativa advierten que en el contexto indígena los factores importantes para el estrés de la crianza tienen que ver con la falta de excedentes económicos, la incertidumbre de trabajo de la madre, la inexistencia de centros de desarrollo y cuidado del niño, el consumo de alcohol del padre, mientras que son protectores del estrés de la crianza el uso de espacios públicos y terrenos amplios, la organización matriarcal y extensa responsable de la seguridad, la inexistencia de inmigrantes, y pavimento, hacen de la vida cotidiana un escenario en el cual las mamás tienen una percepción de seguridad y permiten mayor libertad de movimiento al niño.

Se requiere un modelo de crianza para zonas indígenas que parta del concepto de "conciencia etnica" como el proceso de reproducción social que permite al niño seguir normas, reglas, instrucciones y convertirse en Mayo. Para lograr existen tres variables próximas; a) las afecto-emocional en la interacción familiar que incluya el uso del susto, miedo a través de mitos étnicos y la regulación de afectos para lograr moldear conductas socialmente aceptables; b) observar la relación el niño con el entorno sin restricciones o supresión de riesgos o peligros; c) observación y 
análisis de la inducción al niño en rituales y mitología étnica. Esta triada solo tiene sentido social en un entorno que coloque la lengua de origen en un lugar de privilegio frente a la lengua dominante y la familia y el grupo social se sientan orgullosos de su uso y resguardo.

Estos elementos próximos asociados con la transformación "consciente" del niño dependen de la construcción colectivista de la educación infantil, las características de personalidad de la madre y la participación del padre como proveedor y monitor de los progresos del niño y a su vez la madre como promotora de uso de la lengua, de su escritura y motivadora de su uso en los escenarios social, educativo y cultural.

Lo anterior tiene sentido social cuando la "lengua" como categoría de importancia única y fundamental para volverse "consciente" es amalgamado con la de juego y el niño aprende jugando a hablar y habla para jugar. A través de la tradición oral de la comunidad, de sus gustos, rituales y tradiciones religiosas, culinarias, vestido, vivienda $y$ en fin el mundo Mayo en el que está inmerso, van delineando los caminos de la "conciencia del niño".

La educación inicial en las zonas indígenas debería considerar los siguientes aspectos: cósmica, plurilingüe, intercultural, técnica, científica, comunitaria, práctica, funcional, creativa, crítica, auto gestionaría, solidaria, dinámica, y participativa; lo cual conforma un enfoque pertinente a las condiciones de las poblaciones indígenas de Sonora y de México (Gálvez, 2000). Resulta urgente continuar realizando estudios respecto al servicio de educación inicial en las comunidades indígenas para mejorar este servicio, haciéndolo pertinente y eficaz para las características de cada grupo social, y consecuentemente continuar estudiando los procesos de reproducción social desde diferentes esquemas conceptuales y técnicas metodológicas.

\section{Referencias}

Abidin, R. (1990). Introduction to the special issue: The stress of parenting. Journal of Clinical Child Psychology, 19, 298-301.

Abidin, R. \& Brunner, J. (1995). Development of a Parenting Alliance Inventory. Journal of Clinical Child Psychology, 24, 31-40.

Aguilar, A. (2007). Pueblos indígenas de México, Serie monografías. Obtido do World Wide Web: http://cdi.gob.mx/ini/monografias/mayos.html
Atkin, L. C., Supervielle, T., Sawyer, R. \& Canton, P. (1987). Paso a paso: como evaluar el desarrollo y crecimiento de los niños. UNICEF/PAX. México.

Belsky, S. (1984). The determinants of parenting: a process model. Child development. American Psychologist, 55, 83-96.

Bronfenbrenner, U. (1979). The ecology of buman development. Cambridge, Mass: Harvard, University Press.

Caldwell, B. \& Bradley, R. (1968). Home observations for measurement observations in families of infant, toddlers and preschoolers. USA: University of Arkansas.

Camberos, M., Genesta M. A. \& Huesca, L. (1994). La pobreza en Sonora: los limites a la modernización. Revista de Estudios Sociales, 5(9), 161- 167.

Cunningham, M. (2001). Educación intercultural bilingüe en los contextos multiculturales. Parte III. Universidad de las Regiones Autónomas de la Costa Caribe Nicaragüense. Obtido do World Wide Web: http://www.beps.net/publications/ParteIII.pdf.

Gálvez, I. (2000). La educación inicial en el ámbito internacional: situación y perspectivas en iberoamérica y europa. Revista Iberoamericana de educación. 22, Educación Inicial.

INEGI (2005). Estados Unidos Mexicanos. XII Censo General de Población y Vivienda, 2000. Tabulados Básicos y por Entidad Federativa. Bases de Datos Tabulados de la Muestra Censal. Aguascalientes, Ags., México.

Jaramillo, J., Díaz, O. K. \& Andrea, N. K. (2006). Factores individuales, familiares y escolares asociados a la aceptación y el rechazo social en grupos de niños escolarizados entre los 9 y 11 años de edad. Diversitas, 2(2), 205-215.

Kerlinger, F. \& Lee, H. (2002). Investigación del comportamiento. McGraw-Hill: México.

Laborín, J. F. \& Vera, N. J. A. (2006). Medición de locus de control en dos contextos culturales latinoamericanos. Asociación Mexicana de Psicología Social. (Org.). La Psicología Social en México (pp. 80-86). México: AMEPSO.

León, P. (2005). La llegada del alma. Lenguaje, infancia y socialización entre los mayas de Zinacatán, Instituto Nacional de Antropología e Historia-Centro de Investigaciones y Estudios Superiores en Antropología Social, México.

Martínez, L., Tánori, J. \& Vera, J. A. (2005). Interacción padres e hijos, la estimulación en el hogar y el desarrollo del niño en una zona rural de 
pobreza extrema. VIII Congreso Nacional de Investigación Educativa. Universidad de Sonora. Del 30 de Octubre al 02 de Noviembre.

Peña R. M. O. (2004). Crianza y desarrollo infantil en familias rurales de la región sur del estado de Sonora. Tesis de Maestría en Desarrollo Regional. Hermosillo Sonora, Agosto.

Peña, M., Quihui, A. \& Vera, J. A. (2004) Estrés materno y desarrollo infantil en comunidades del Estado de Sonora. Em: E.A. Carlos-Martínez, J. E. Ramos-Salas \& L. A. Galván-Parra. Anuario de Investigaciones Educativas (pp. 171-180). Red de Investigaciones Educativas.

Peña, R. M. O., Aguilar, R. C. \& Vera, N. J. A. (2005). Pareja, estimulación, y desarrollo del infante en zona rural en pobreza extrema. Revista Mexicana de Investigación Educativa, 10(25), 559-576.

Pérez-Gómez, G. (2008). Crianza y desarrollo infantil en familias Yoreme/mayo en el norte de Sinaloa. Tesis. Mención Honorífica. Universidad Autónoma Indígena de México.

PNUD. (2001). El desarrollo humano: pasado, presente y futuro. El Informe sobre el Desarrollo Humano. Madrid: Mundi-Prensa.

Rojas, C. D. (2002). El conflicto entre tradición y modernidad. Constitución de la identidad cultural indigena Bribri. Obtido do World Wide Web: http://www.flacso.or.cr/fileadmin/documentos/ FLACSO/Cuaderno126.pdf.

Secretaria de Educación Pública - SEP. (2004). Carpeta de Información Básica de Educación Indígena, Sonora. Estadística Básica. Obtido do World Wide Web: http://www.sep.gob.mx/res/sep/sep_SON/7720 ?op $=2 \&$ page $=0$

Sen, A. (2000) Desarrollo y libertad. Barcelona: Planeta S. A.

SEP-CONAFE (1999). Programa de educación inicial no escolarizada. Manual del promotor educativo. México: Impresiones Gráficas de Arte Mexicano.

Shaffer David R. (2000). Psicología del desarrollo: infancia y adolescencia. Mexico: Internacional Thomson Editores.

Super, C. \& Harkness, S. (1987). The developmental niche: a conceptualization at the interface of child and culture. International Journal of Behavioral Development, 9, 1-25.

Vera, A., Grubits, S. \& Rodríguez, C. (2007). Estimulación y practicas de crianza en infantes terena del Brasil. Revista Ra Ximbai, 3(1), 49-81.
Vera, J. A. (2000). Estudio para ajustar una medida de lectoescritura y matemáticas para escuelas rurales unitarias en el Estado de Sonora, México. Revista Sociedad Académica.

Vera, J. A. (2006) Indigenismo y exclusión. Revista Ra Ximbai, 2(3), 677-681.

Vera, N. J. A. (2003). Manual psicopedagógico para el maestro de educación primaria. CIAD, SEC Sonora, SIMAC y CONACYT. En prensa.

Vera, N. J. A. (2004). Desarrollo regional e indicadores de desarrollo humano. Revista de Estudios Sociales, 12(24), 181-188.

Vera, N. J. A., Calderón, G. N. \& Torres, A. M. (2007). Practicas de crianza y estrés de la madre en la Etnia Yoreme-Mayo. Estrés de la crianza. Estudios y propuestas para el medio rural, Tomo II, 1-30.

Vera, J. A., Montiel, M., Serrano, E. y Velasco, F. (1997) Objetivos de la crianza, desarrollo, estimulación y sistemas de enseñanza. Psicología y Salud. Instituto de Investigaciones Psicológicas de la Universidad Veracruzana. Nueva Época. Julio-Diciembre. 10, 27-35.

Vera, N. J. A. \& Montiel, C. M. M. (1998). Análisis de las propiedades psicométricas del índice de estrés de la crianza en una población rural del Estado de Sonora. La Psicología Social, México: AMPESO.

Vera, N. J. A., Velasco, A. F. \& Morales, N. D. (2000). Estudio comparativo de familias urbanas y rurales: desarrollo y estimulación del niño. Familia: naturaleza amalgamada. 309-324.

Vígano, D. \& Díaz-Loving, R. (1990). Desarrollo y análisis psicométrico del EMACM: Escala Multidimensional de Autoritarismo en la Cultura Mexicana. Revista Interamericana de Psicología, 24(2), 139-158.

Villegas, M. (2000). La familia y su relación con la crianza. La crianza humanizada. Boletín del grupo de puericultura de la Universidad de Antioquia. Obtido do World Wide Web: http://www.monografias.com/trabajos/preescola res.

Webster-Stratton, C. (1990). Stress: a potential disruptor of parent perceptions and family interactions. Journal of Clinical Child Psychology, 19, 302-312.

Recebido em maio de 2010 Reformulado em julbo de 2010 Aprovado em agosto de 2010 
Sobre os autores:

José Ángel Vera Noriega posee Doctorado en Psicología Social por la Universidad Nacional Autónoma de México. Miembro del Sistema Nacional de Investigadores (Nivel II) y la Academia Mexicana de Ciencias. Actualmente desarrolla investigación con financiamiento nacional e internacional público y privado fundamentalmente con poblaciones rurales e indígenas en los estados del noroeste mexicano en aspectos relacionados con salud y educación.

Claudia Karina Rodríguez Carvajal posee Licenciatura en Psicología por la Universidad de Sonora y Maestría en Desarrollo Regional por el Centro de Investigación en Alimentación y Desarrollo A.C. Especialista en Desarrollo Rural y Educación. Se desempeña como asesora y consultora independiente en proyectos de investigación en Instituciones de Educación Superior.

José Pablo Siqueiros Aguilera es Licenciado en Psicología por la Universidad de Sonora. Estudiante del Posgrado en Desarrollo Regional en el Centro de Investigación en Alimentación y Desarrollo, A.C. Ha participado durante cuatro años en el diseño, obtención y análisis de datos en diferentes estudios sobre Crianza.

Miguel Angel Torres Ávila es Licenciado en Psicología por la Universidad de Sonora y Maestro en Desarrollo Regional por el Centro de Investigación en Alimentación y Desarrollo, A.C., forma parte del equipo de investigación sobre prácticas de crianza desde el año 2003. Actualmente se encuentra trabajando en CEPAVI como facilitador del grupo de reeducación para agresores de violencia de pareja. 
